Document downloaded from:

http://hdl.handle.net/10251/64699

This paper must be cited as:

Sonkki, M.; Sánchez Escuderos, D.; Hovinen, V.; Salonen, E.; Ferrando Bataller, M. (2015). Wideband Dual-Polarized Cross-Shaped Vivaldi Antenna. IEEE Transactions on Antennas and Propagation. 63(6):2813-2819. doi:10.1109/TAP.2015.2415521.

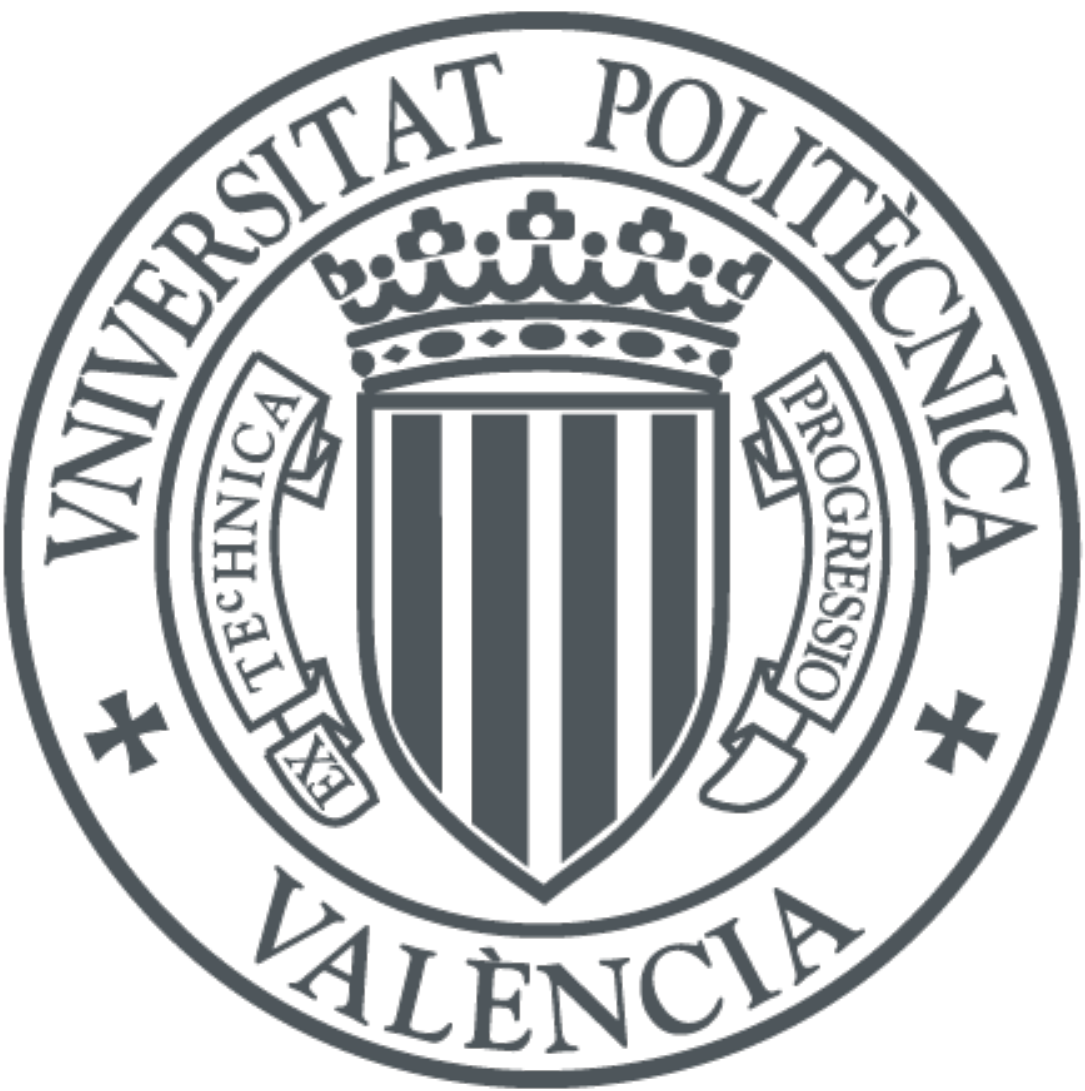

The final publication is available at

http://dx.doi.org/10.1109/TAP.2015.2415521

Copyright Institute of Electrical and Electronics Engineers (IEEE)

Additional Information 


\section{Wideband Dual-Polarized Cross-Shaped Vivaldi Antenna}

\author{
Marko Sonkki, Daniel Sánchez-Escuderos, Veikko Hovinen, \\ Erkki T. Salonen, Miguel Ferrando-Bataller
}

\begin{abstract}
This paper presents a wideband, dual-polarized Vivaldi antenna or tapered slot antenna with over a decade (10.7:1) of bandwidth. The dual-polarized antenna structure is achieved by inserting two orthogonal Vivaldi antennas in a cross shaped form without a galvanic contact. The measured -10 dB impedance bandwidth $\left(S_{11}\right)$ is approximately from $0.7 \mathrm{GHz}$ up to $7.30 \mathrm{GHz}$, corresponding to a $166 \%$ relative frequency bandwidth. The isolation $\left(S_{21}\right)$ between the antenna ports is better than $30 \mathrm{~dB}$, and the measured maximum gain is 3.8-11.2 dB at the aforementioned frequency bandwidth. Orthogonal polarizations have the same maximum gain within the $0.7-3.6 \mathrm{GHz}$ band, and a slight variation up from 3.6 GHz. The cross polarization discrimination (XPD) is better than $19 \mathrm{~dB}$ across the measured 0.7-6.0 GHz frequency bandwidth, and better than $25 \mathrm{~dB}$ up to 4.5 GHz. The measured results are compared with the numerical ones in terms of $S$-parameters, maximum gain, and XPD.
\end{abstract}

Index Terms - Antenna Measurements, MIMO OTA Test System, Tapered Slot Antenna, Ultra Wideband Antenna, UWB

\section{INTRODUCTION}

A traditional way to implement directive wideband antennas is to use linearly polarized double-ridge horn antennas [1]-[4] or dual-polarized quad-ridge horn antennas [5]-[8]. Another way to implement a wideband dual-polarized antenna is to orientate two tapered slot antennas, also called Vivaldi antennas, into a cross-shape with respect to the antenna center, previously studied in [9]-[13], where a galvanic contact is avoided by a small longitudinal gap between antenna elements. To the authors' best knowledge, a cross-shaped Vivaldi antenna structure is not very commonly studied in the literature when considering two orthogonal polarizations.

The most common way to implement two orthogonal polarizations with Vivaldi antennas is to place them orthogonally along the outer edge of each element [14]-[16]. In [16] a case has been reported where with linear and dual-polarized Vivaldi antenna arrays, a $170 \%$ relative $-10 \mathrm{~dB}$ impedance bandwidth has been achieved, whereas [14] reports $164 \%$ relative $-6 \mathrm{~dB}$ impedance bandwidth.

Manuscript received 4nd April 2014. This work was supported by the Finnish Funding Agency for Technology and Innovation (Tekes, project MIMOTA) and its industrial partners, Nokia Devices Oulu, Pulse Finland, EB, and ETS Lindgren, and by the Spanish Ministry of Economy and Competitiveness under the projects TEC2010-20841-C04-01 and CSD2008-00068. Mr. Sonkki also would like to thank the Nokia Foundation and Infotech Oulu Doctoral Program for financially supporting his PhD studies. This technology has been licensed by industry.

M. Sonkki, V. Hovinen and E. T. Salonen are with the Centre for Wireless Communications (CWC), P.O.B. 4500, FI-90014, University of Oulu, Finland (e mail: marko.sonkki@ee.oulu.fi).

D. Sánchez-Escuderos and M. Ferrando-Bataller are with the Institute of Telecommunications and Multimedia Applications (iTEAM), Edificio 8G, Universitat Politècnica de València, Camino de Vera s/n, 46022, Valencia, Spain (e mail: dasanes1@iteam.upv.es).

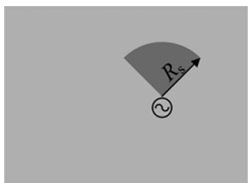

(a)

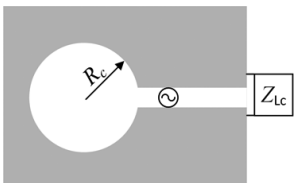

(b)

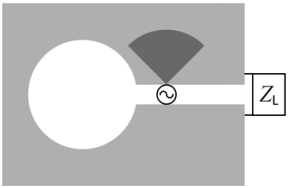

(c)
Fig. 1. Studied equivalent circuit components: (a) stub, (b) cavity, and (c) combination of stub and cavity representing the Vivaldi feeding.

This paper presents a wideband dual-polarized Vivaldi antenna. The goal of the antenna design was to find an affordable antenna structure with good antenna characteristics over a wide frequency range. The starting point of the antenna design was to find a suitable dual-polarized antenna structure for MIMO OTA (Multiple-Input-Multiple-Output Over-The-Air) test system [10] to cover multiple wireless standards under one measurement system. As a comparison, multiple wireless standards can be covered, e.g. by using a quad-ridged dual-polarized horn antenna, as presented in [11]. The development of a MIMO OTA test system is under investigation at the moment in industrial companies and scientific communities.

Compared to cross-shaped dual-polarized Vivaldi antenna structures presented in [12] and [13], the antenna structure proposed in this paper offers a wider relative operating bandwidth. The proposed antenna structure has $168 \%$ relative $-8 \mathrm{~dB}$ impedance bandwidth and $166 \%$ relative $-10 \mathrm{~dB}$ impedance bandwidth, whereas [12] presents $51 \%(-8 \mathrm{~dB})$ and [13] $110 \%(-10 \mathrm{~dB})$. The isolation between the feeding ports of the proposed antenna is better that $30 \mathrm{~dB}$. As a comparison, the cross-shaped Vivaldi antenna presented in [13] offers isolation between $20 \mathrm{~dB}$ and $30 \mathrm{~dB}$.

The proposed dual-polarized Vivaldi antenna has excellent radiation properties in terms of measured total efficiency, with a variation between -1.75 and $-0.05 \mathrm{~dB}$ within the measured $0.7-6 \mathrm{GHz}$ bandwidth. On the other hand, the measured maximum gain at boresight is between 3.8-11.2 dB with XPD (cross polarization discrimination) better than $19 \mathrm{~dB}$ at the aforementioned bandwidth. For comparison, in [13] the measured XPD over the operating bandwidth is approximately $15 \mathrm{~dB}$.

The paper is organized as follows: Section II introduces an equivalent circuit study of the balanced feed mechanism, and Section III describes the proposed dual-polarized wideband Vivaldi. In Section VI, the Vivaldi antenna is investigated in terms of measured $S$-parameters and radiation properties. Finally, Section V contains the conclusions and a short discussion of the proposed antenna.

\section{Equivalent CiRCUIT Simulations OF FEed MECHANISM}

The Vivaldi antenna is a balanced antenna structure. Thus, the structure needs a balun to transfer or receive the electromagnetic waves properly over the tapered slot. The most common balun structure used with Vivaldi antennas is adapted from Knorr's microstrip-to-slot transition [17].

To study the characteristics of the balanced feeding over a wide frequency range, the balanced feed structure is separated 

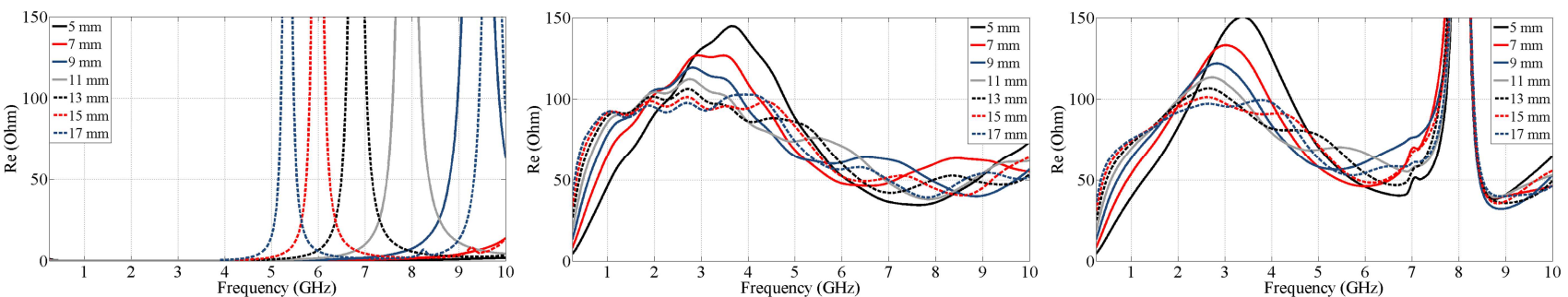

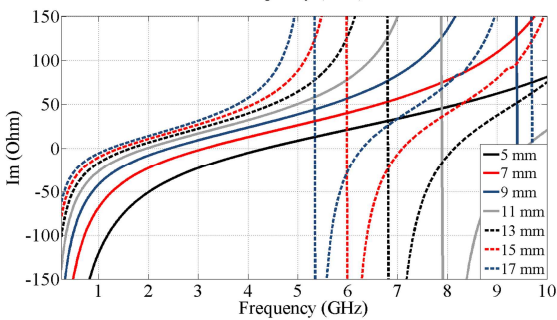

(a)

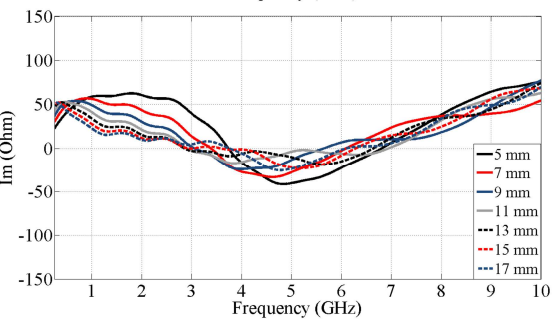

(b)

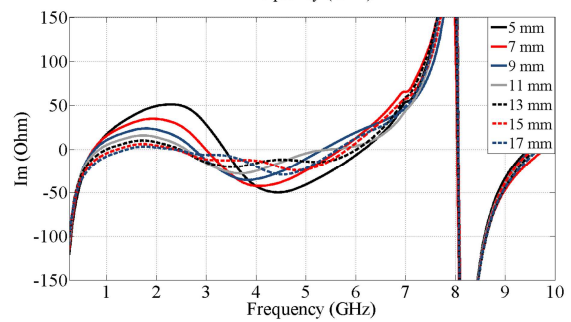

(c)

Fig. 2. Simulated input impedance of (a) stub with variable stub radius $R_{\mathrm{s}}$, (b) cavity with variable cavity radius $R_{\mathrm{c}}$, and (c) the combination of stub and cavity with fixed $R_{\mathrm{s}}=11 \mathrm{~mm}$ and variable cavity radius $R_{\mathrm{c}}$.

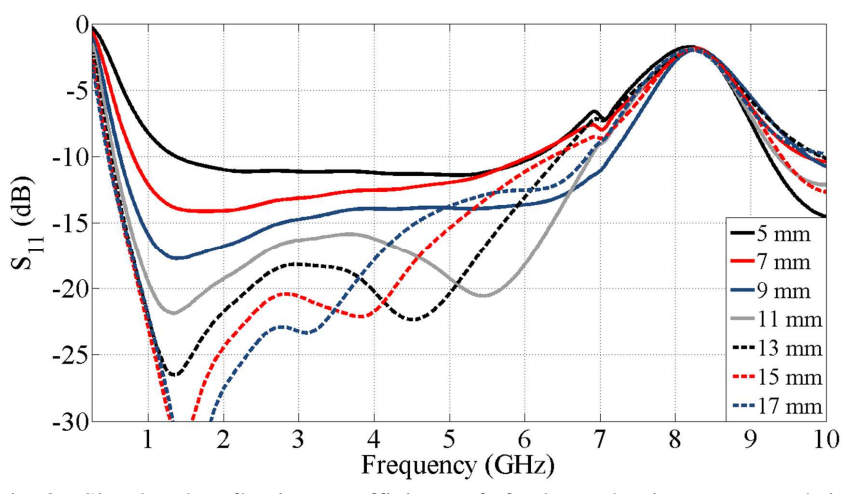

Fig. 3. Simulated reflection coefficient of feed mechanism presented in Fig. 1(c). Radius of the stub is fixed $R_{\mathrm{s}}=11 \mathrm{~mm}$, when the cavity radius $R_{\mathrm{c}}$ is swept from $5 \mathrm{~mm}$ to $17 \mathrm{~mm}$ in $2 \mathrm{~mm}$ steps.

into three equivalent circuit parts as presented in Fig. 1. These parts are (a) a stub, (b) a cavity, and (c) a combination of stub and cavity representing the feed mechanism of the proposed Vivaldi antenna structure. The substrate selected for the equivalent circuits is Rogers 4003 RF-laminate $\left(\varepsilon_{\mathrm{r}}=3.38\right)$, which is the same as the one used in the proposed antenna.

In Fig. $1, R_{\mathrm{s}}$ and $R_{\mathrm{c}}$ are the radius of the stub and the cavity, respectively, whereas $Z_{\mathrm{Lc}}$ and $Z_{\mathrm{L}}$ are the loads to match the open ended slot to the free-space impedance. In this study, $Z_{\mathrm{Lc}}=Z_{\mathrm{L}}=85 \Omega$, which is the average input impedance (from $0.5 \mathrm{GHz}$ to $10 \mathrm{GHz}$ ) of the isolated Vivaldi antennas fed by a slot transmission line. Even though an average value is applied, results with constant impedance are more valuable than ending the slot transmission line with an open circuit.

The equivalent circuits of Fig. 1 have been simulated with CST Microwave Studio [18] for a range of $R_{\mathrm{s}}$ and $R_{\mathrm{c}}$ values between $5 \mathrm{~mm}$ and $17 \mathrm{~mm}$, and a stub angle of $80^{\circ}$. Fig. 2 (a) presents the input impedance (real and imaginary parts) of the stub shown in Fig. 1 (a) as a function of frequency. As can be observed, the real part of the stub is zero until the stub reaches anti-resonance [19]. At this frequency, the behavior changes, the stub becomes an open circuit, and the imaginary part behavior changes from inductive to capacitive. Thereby, the anti-resonance frequency limits the upper band of the feed structure.

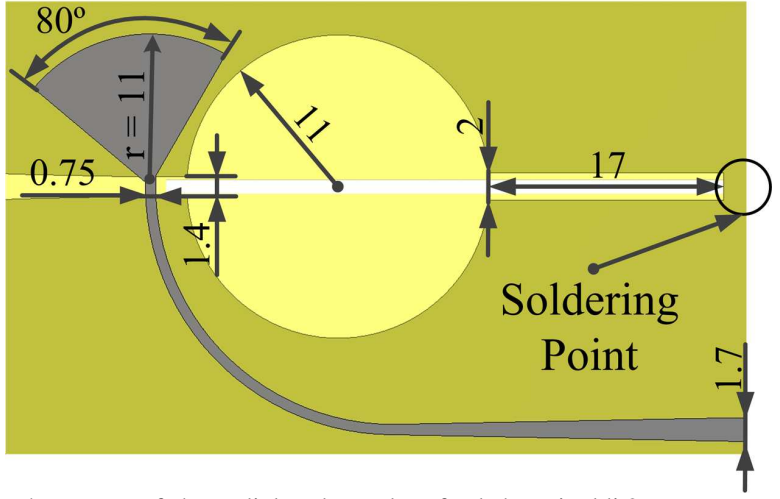

Fig. 4. Layout of the radial stub used to feed the Vivaldi 2 antenna. Both Vivaldis have the same feeding mechanism. Dimensions are in $\mathrm{mm}$.

Fig. 2 (b) presents the simulation results of a cavity with the same radius as the stub studied in Fig. 2 (a). The imaginary part of the cavity stays quite constant over the bandwidth, and presents a small variation when increasing the radius. The wide bandwidth and the small variation of the imaginary part can be explained by considering the cavity of Fig. 2 (b) as a quarter-wavelength slot line ended in a short-circuit. Since a wide circular slot line is used, instead of a narrow quadrangular slot line [17], the bandwidth of the cavity can be maximized.

Additionally, the real part shows more variation. When the cavity radius increases, the real part of the input impedance rapidly changes at the lower frequencies and stays more constant as the radius and frequency increases. The smaller the cavity radius is, the more variation the real part presents across the bandwidth.

Fig. 2 (c) presents the combined results of the real and imaginary parts of the stub and the cavity. In this study the stub radius is kept constant $\left(R_{\mathrm{S}}=11 \mathrm{~mm}\right)$, and the cavity radius is swept as in Fig. 2 (a) and (b). It can be clearly seen how the characteristics of the real and imaginary parts are well summarized. The real part represents mainly the characteristics of the cavity. On the other hand, the imaginary parts, especially with the larger cavity sizes, are summarized as complementary offering impedance variation around zero. Also, it can be 


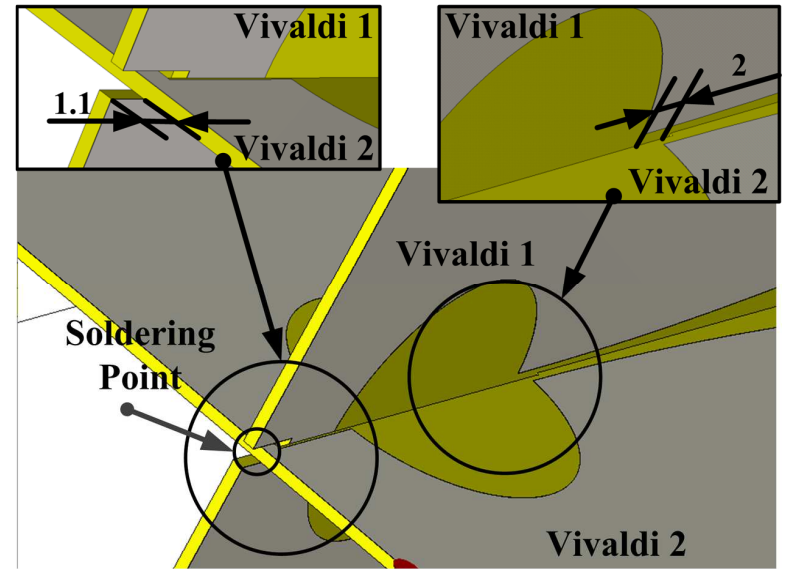

Fig. 5. Layout of the cross-shaped dual-polarized Vivaldi antenna feeding. Dimensions are in $\mathrm{mm}$.

observed how the stub anti-resonance defines the upper frequency limit of the feed structure.

Fig. 3 presents the combined study of the reflection coefficient of the stub and cavity, shown in Fig. 1 (c). It can be observed that, when the cavity radius equals to $9 \mathrm{~mm}$ and $13 \mathrm{~mm}$, the frequency response also presents a good matching. Still, the overall performance with $R_{\mathrm{s}}=R_{\mathrm{c}}=11 \mathrm{~mm}$ gives the best performance. Note that results of Fig. 3 do not represent the return loss of the proposed structure (feeding circuit connected to a Vivaldi antenna), which will be provided later, but they are an approximation of the results in the final structure. This difference is caused by the use of an average load instead of the real input impedance of the Vivaldi antenna at the different studied frequencies.

Thus, it can be concluded that the cavity determines the behavior of the feed, and the stub the upper frequency limit. In addition, results show that a cavity and a stub with the same radius $(11 \mathrm{~mm})$ offer the best impedance matching over the desired frequency range.

The presented study offers a fast way to design a wideband balanced feeding and gives the designer a tool to adapt the antenna structure between the antenna feed and the free-space impedance. However, the characteristic impedance of the transmission line between the antenna port and the stub needs to be designed separately based on slot width, and this study does not take that into account. Also it is good to mention that this is a general study and does not include for example the slot behind the cavity of Vivaldi 2 and the position of the feeding point.

\section{Prototype Antenna Structure}

This section presents the prototype of the proposed dual-polarized cross-shaped Vivaldi antenna. First, the antenna feed, identical for both Vivaldi antennas, is introduced with its main dimensions. Then, the implementation of the dual-polarized feeding is presented by combining both Vivaldi antennas together in a single structure. Finally, the photographs of the prototype antenna are presented and discussed. Commercial simulator CST Microwave Studio [18] has been used to simulate the antenna.

\section{A. Feeding Mechanism}

Figure 4 presents the feeding mechanism used to feed both

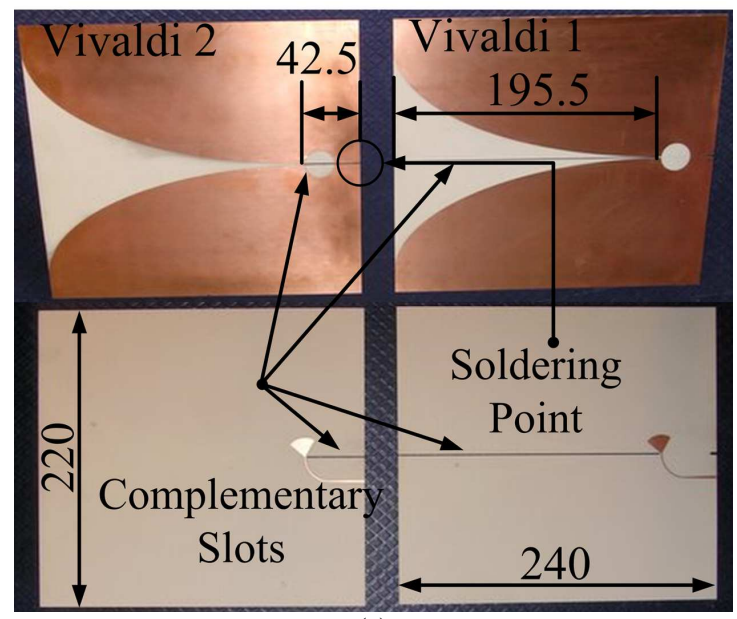

(a)

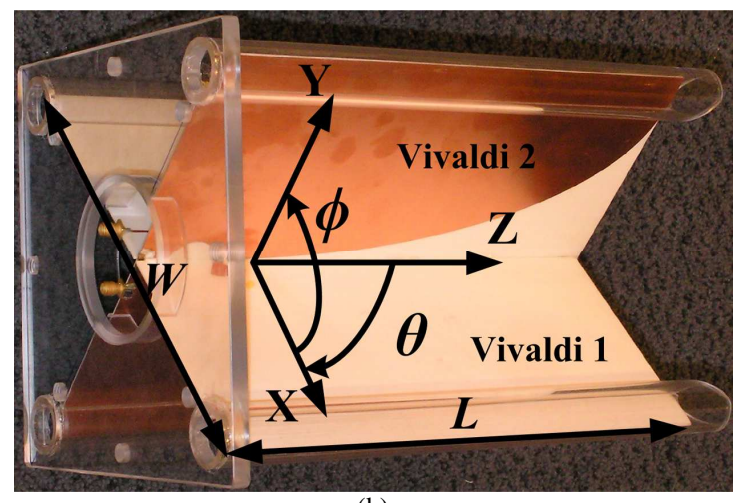

(b)

Fig. 6. Photograph of (a) individual Vivaldi element on both sides, and (b) the wideband dual-polarized Vivaldi antenna with coordinate system and antenna support.

Vivaldi antennas in the proposed structure. The feeding is implemented with a radial stub performing the impedance matching from $50 \Omega$ to approximately $85 \Omega$, as described in Section II. An SMA connector is used to deliver the signal to the antenna. The $17 \mathrm{~mm}$ slot in the substrate is used to make possible the overlapping of the orthogonal Vivaldi antennas, labeled as Vivaldi 1 and Vivaldi 2 (see Fig. 5).

As concluded in Section II, the optimal cavity and stub radius are $11 \mathrm{~mm}$, with a stub angle of $80^{\circ}$. These values provide the maximum impedance bandwidth around $7.3 \mathrm{GHz}$ for the average input impedance of the Vivaldi antennas.

\section{B. Dual-Polarized Prototype Antenna Structure}

This section shows the implementation of two orthogonal Vivaldi antennas without galvanic contact. The principal characteristics of the implementation are presented in Fig. 5. Rogers 4003 RF-laminate $\left(\varepsilon_{\mathrm{r}}=3.38\right)$ is used to fabricate the antenna. The same radial stub described in previous section is used to feed both Vivaldi antennas.

Fig. 5 shows how Vivaldi antennas are overlapping each other so that Vivaldi 2 is $2 \mathrm{~mm}$ ahead of Vivaldi 1 in the $\mathrm{Z}$ axis. This $2 \mathrm{~mm}$ shift is done to avoid the antenna feeding lines overlapping each other. In order to compensate the displacement of the phase center of the separate antennas in the $\mathrm{Z}$ axis, the microstrip feed line of Vivaldi 1 is $1.1 \mathrm{~mm}$ shorter (see Fig. 5), corresponding to approximately $2 \mathrm{~mm}$ difference 


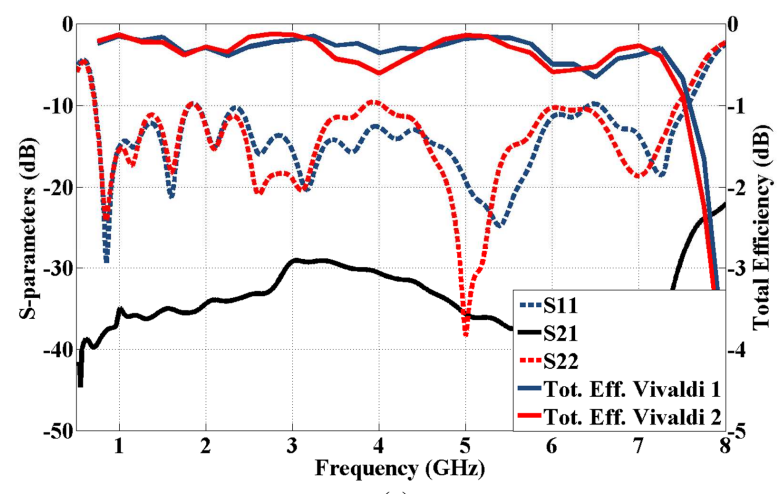

(a)

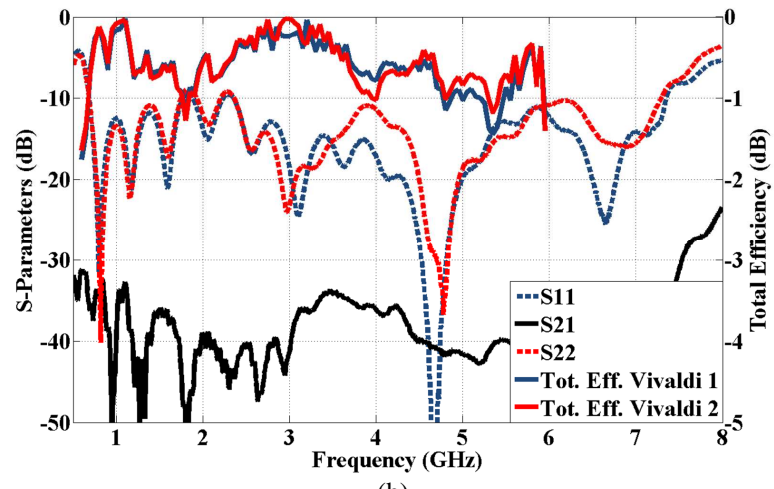

(b)

Fig. 7. (a) Simulated and (b) measured $S$-parameters and total efficiency of the dual-polarized Vivaldi antenna.

in free space.

A photograph of the individual Vivaldi elements in the dual-polarized structure is presented in Fig. 6 (a). The lengths of the complementary slots shown in the figure are $42.5 \mathrm{~mm}$ and $195 \mathrm{~mm}$. This allows the overlapping implementation of the orthogonal Vivaldi elements. Note that the summarized length of the slots is $238 \mathrm{~mm}$, which corresponds to the $2 \mathrm{~mm}$ displacement.

The studied cross-shaped dual-polarized Vivaldi prototype antenna is presented in Fig. 6 (b) together with the considered coordinate system. The antenna has a length $L=240 \mathrm{~mm}$ and width $W=220 \mathrm{~mm}$, and its tapered slot opens elliptically outward from the feeding point.

A high opening rate of the Vivaldi improves the resistance at low frequencies but increases the complex impedance variation as the frequency increases. A compromise is required by 3D simulations when choosing the opening rate and cavity size to obtain the desired antenna performance. An elliptical form suppresses reflections from the antenna opening at low frequencies [15], [20].

The complementary slots (Fig. 6 (a)) are cut into the center line of the antenna structures to allow the overlapping and keeping antennas isolated without galvanic contact. The width of the complementary slots is $1 \mathrm{~mm}$, and it is slightly wider than the substrate thickness $(0.813 \mathrm{~mm})$.

In order to implement two orthogonal Vivaldi antennas in a cross shaped form, the slot behind the cavity must be first opened (Fig. 6a) to allow Vivaldi 2 to slide along the complementary longitudinal slot of Vivaldi 1 in direction $\mathrm{Z}$. The galvanic connection between the two halves of Vivaldi 2 is returned by soldering a piece of conducting material over the opened slot. The soldering point is marked in Fig. 4, 5 and 6.

When comparing the dual-polarized feeding mechanism to the one presented in [13], Vivaldi 2 is ahead of Vivaldi 1, like in the antenna proposed in this paper, but there is no cut behind the cavity. This cut in the cavity of the proposed antenna makes possible the implementation of the feeding lines close to each other, although imposes a small shift between the phase centers of both antennas. This shift, which might lead to severe problems when considering circular polarization, can be corrected by using feeding lines with different lengths, as commented above.

\section{MEASUREd PRototype ANTENNA}

This section presents the measurement results of the dual-polarized Vivaldi prototype antenna. The antenna characteristics are studied in terms of $S$-parameters, total efficiency, and radiation patterns at two different center frequencies. Side lobe level, $3 \mathrm{~dB}$ beamwidth, maximum gain, and cross polarization discrimination (XPD) are presented as a function of frequency. The maximum gain and the XPD are shown at boresight direction $\left(\theta=0^{\circ}\right)$.

\section{A. S-parameters}

Fig. 7 presents the simulated and measured $S$-parameters and total efficiencies of the dual-polarized Vivaldi prototype antenna. The measured $-10 \mathrm{~dB}$ impedance bandwidth is $683 \mathrm{MHz}-7.30 \mathrm{GHz}$, which corresponds to a $166 \%$ relative $-10 \mathrm{~dB}$ impedance bandwidth. This well correlates with the relative bandwidth of the Vivaldi array presented in [16], where $170 \%$ relative $-10 \mathrm{~dB}$ bandwidth was achieved. Note that the measured matching does not fully satisfy the $-10 \mathrm{~dB}$ level at 1.8 and $2.3 \mathrm{GHz}$. At those frequencies, the matching is $-9 \mathrm{~dB}$. This higher level, as well as the small frequency shift in some dips of the frequency response, is mainly caused by manufacturing imperfections and the use of an average electrical characterization of the substrate for the whole bandwidth. Nevertheless, apart from these differences, a reasonably good correlation between the measured and simulated $S$-parameters are observed in Fig. 7.

The simulated and measured mutual coupling between the antenna ports is lower than -29 and $-30 \mathrm{~dB}$, respectively, and predicts good polarization properties. Furthermore, the measured total efficiency is between -1.75 and $-0.05 \mathrm{~dB}$ within 0.7-6 GHz bandwidth. The total efficiency was measured in a narrower bandwidth than the $S$-parameters by the Satimo near-field antenna measurement system [21]. The simulations predicted a slightly higher total efficiency, between -0.61 and $-0.13 \mathrm{~dB}$, over the aforementioned frequency band than the measured one. The difference is explained by the measurement tolerance and slightly different loss coefficients in the simulation model compared to the measured prototype.

For comparison, in [13] the measured isolation between antenna ports is between $20 \mathrm{~dB}$ and $30 \mathrm{~dB}$, and, thus, presented results are better than the compared results in the literature. As a conclusion, the antenna structure proposed in this paper is well matched over the measured frequency band, with good 

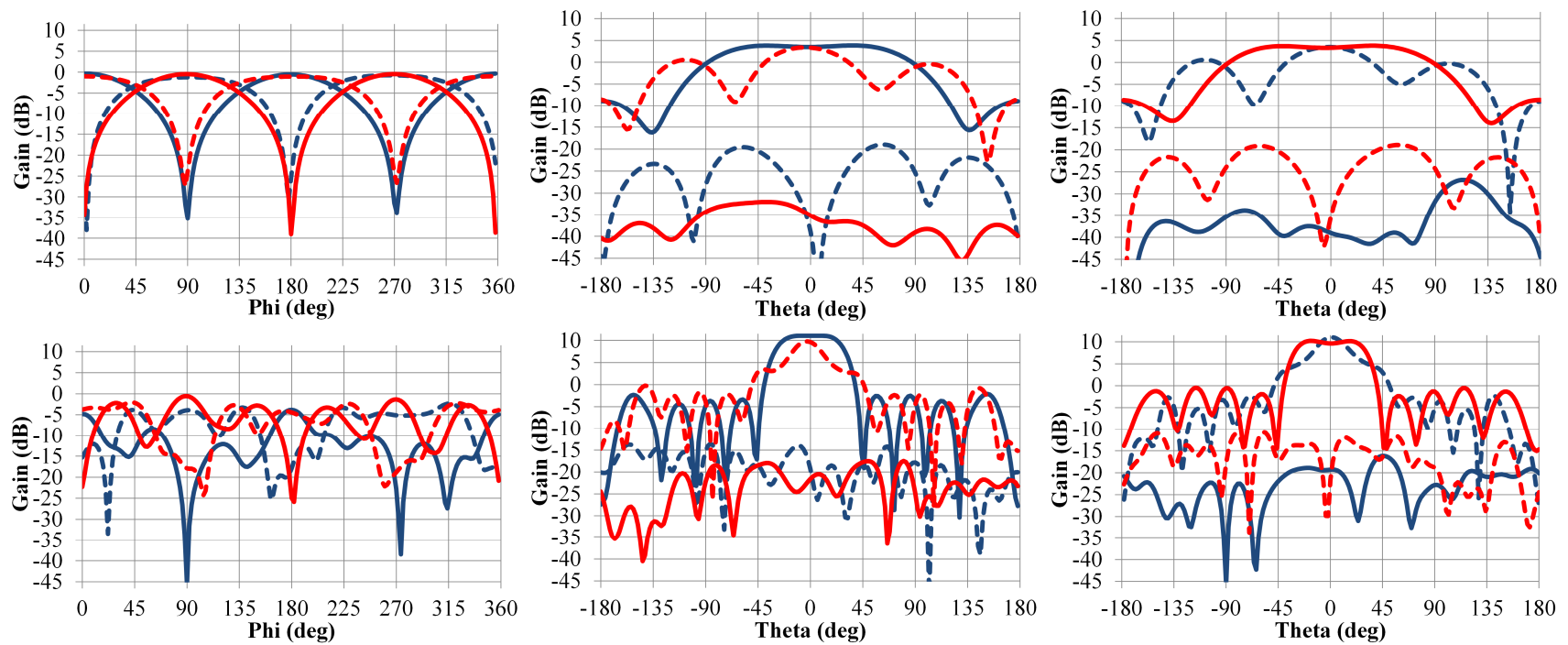

Fig. 8. Measured radiation patterns at $700 \mathrm{MHz}$ (upper row) and $3 \mathrm{GHz}$ (lower row). The legend of the lines is as: (- -) Vivaldi 1 - Phi; (- - -) Vivaldi 1 - Theta; (-) Vivaldi 2 - Phi; and (- - ) Vivaldi 2 - Theta. The cuts go from left to right in the following order: XY, XZ, YZ.

total efficiency and isolation between the antenna ports.

\section{B. Radiation Properties}

Fig. 8 presents the radiation patterns of the Vivaldi antenna prototype at 0.7 and $3 \mathrm{GHz}$. The patterns are presented in XY-, $\mathrm{XZ}$-, and YZ-cuts, and every cut includes both polarization components (Phi and Theta). In the measurements, the Vivaldi 2 was terminated with a standard $50 \Omega$ load when the total efficiency of Vivaldi 1 was measured, and vice versa. The measurements were done with Satimo near-field antenna measurement system [21].

It can be seen how both antennas have similar radiation properties; the Phi-component of Vivaldi 1 is equal to the Theta-component of the Vivaldi 2, and vice versa. This is due to the orthogonal orientation of the antennas. In addition, the higher the frequency, the narrower the main lobe of the antenna becomes. This is due to the fact that when the wavelength gets smaller related to the antenna size, the directivity increases.

Also, the number of side lobes increases at higher frequency because current nulls start to appear in the antenna structure. This increases the number of nulls in the radiation pattern as higher-order modes are excited. In Fig. 8, the difference between the measured main lobe and side lobe level is between

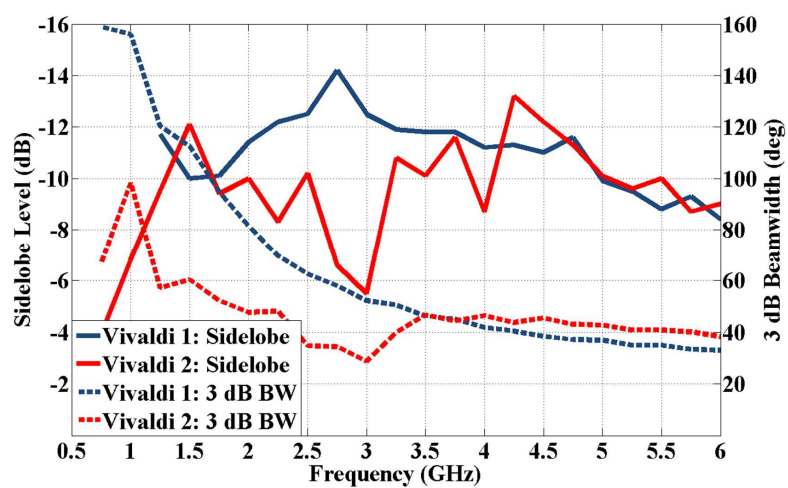

Fig. 9. The simulated side lobe level and $3 \mathrm{~dB}$ beamwidth as a function of frequency. Both parameters are presented in YZ-cut to get an overall picture of functionality of the cross-shaped Vivaldi antenna.

\section{5-15 dB, depending on the operating frequency.}

Fig. 9 presents the simulated side lobe levels and $3 \mathrm{~dB}$ beamwidths as a function of frequency of the proposed antenna structure. For simplicity, both parameters are presented only in YZ-cut as the radiation properties of Vivaldis are close to symmetrical and, hence, parameters in the XZ-cut are quite similar (i.e., XZ-cut of Vivaldi 1 is similar to the YZ-cut of Vivaldi 2 and vice versa), as shown previously (Fig. 8). Side lobe level here is the ratio of the power density in the side lobe to the power density in the main lobe. Side lobe level for Vivaldi 1 starts at $1.25 \mathrm{GHz}$ as the simulations did not predict side lobes at lower frequencies. The side lobe levels are varying between -14 and $-6 \mathrm{~dB}$, whereas the $3 \mathrm{~dB}$ beamwidth is between $35^{\circ}$ and $160^{\circ}$.

Fig. 10 presents the simulated and measured maximum gain as a function of frequency up to $6 \mathrm{GHz}$ for both antennas. The frequency band is limited to $6 \mathrm{GHz}$ by the measurement system as discussed above. The measured maximum gain at boresight $\left(\theta=0^{\circ}\right)$ is between $3.8-11.2 \mathrm{~dB}$, whereas simulation predicts 4.3-10.9 $\mathrm{dB}$ at the 0.7-6.0 GHz frequency bandwidth. Both have relatively the same gain up to $3.6 \mathrm{GHz}$. Also, it can be noticed that, on the one hand, there exist a difference smaller than $1.5 \mathrm{~dB}$

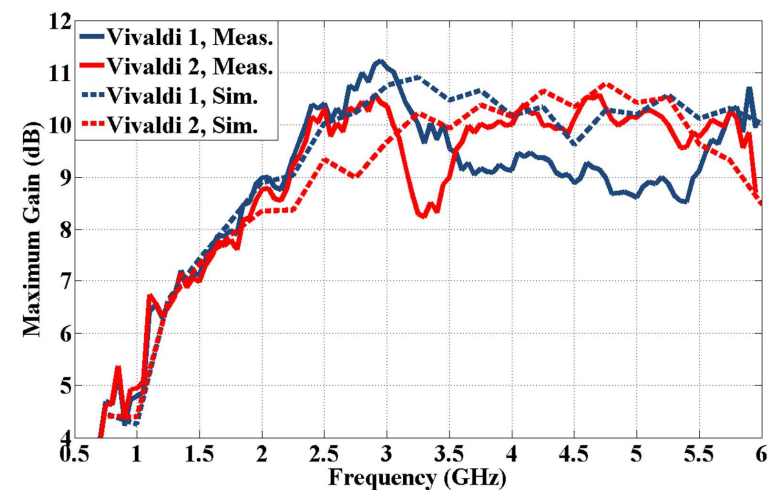

Fig. 10. Simulated and measured maximum gain of the Vivaldi 1 and Vivaldi 2 as a function of frequency. 


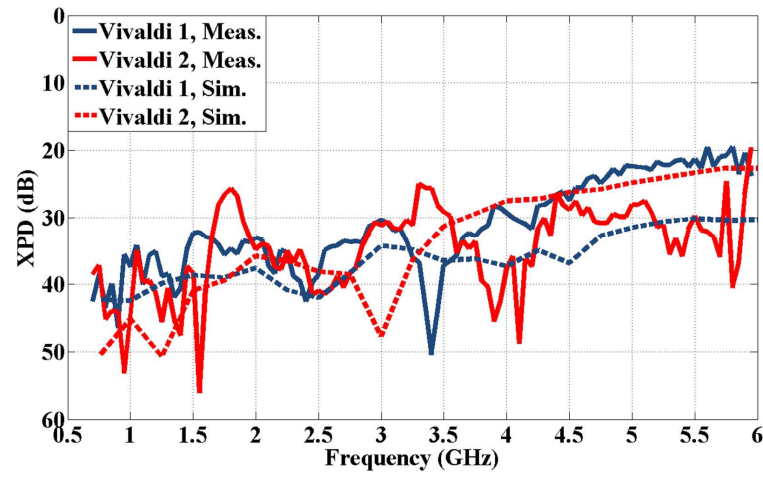

Fig. 11. Measured XPD of the Vivaldi 1 and Vivaldi 2 as a function of frequency in boresight direction $\left(\theta=0^{\circ}\right)$.

in the maximum gain between the Vivaldi antennas from $3.6 \mathrm{GHz}$ to $6 \mathrm{GHz}$. This gap is mainly caused by different manufacturing errors in the two Vivaldi antennas, which also caused differences in the measured S11 parameter. On the other hand, the variation of the measurement is smaller than $2 \mathrm{~dB}$, whereas the simulation predicts a variation smaller than $1 \mathrm{~dB}$. The maximum gain follows the trend presented in [9] and [13].

Fig. 11 presents the simulated and measured antenna performance in terms of XPD as a function of frequency in the direction of boresight $\left(\theta=0^{\circ}\right)$. As it can be observed, the difference between the polarization components is better than $25 \mathrm{~dB}$ up to $4.5 \mathrm{GHz}$. After $4.5 \mathrm{GHz}$ the XPD is below $20 \mathrm{~dB}$ around $6 \mathrm{GHz}$, which can be still considered good. As a comparison, the XPD in [9] and [13] is approximately $15 \mathrm{~dB}$ within the operating frequency band.

As a conclusion, the dual-polarized Vivaldi antenna has good radiation characteristics over a wide frequency bandwidth. Some small changes in the maximum gain and XPD of the individual antenna elements can be observed. This might be caused by the overlapping of the antennas, which produces a non-symmetrical structure.

\section{CONCLUSION AND DISCUSSION}

A wideband dual-polarized Vivaldi antenna has been presented with $166 \%$ relative $-10 \mathrm{~dB}$ impedance bandwidth from $683 \mathrm{MHz}$ to $7.30 \mathrm{GHz}$. The antenna consists of two orthogonally orientated Vivaldi antenna elements in a cross form. The structure has good antenna performance in terms of the measured impedance bandwidth and radiation properties.

The proposed antenna is presented to be used in a MIMO OTA test system. Nevertheless, the dual-polarized antenna might be also useful in other kind of antenna measurement systems. An especially important application might be in systems where both polarizations need to be measured at the same point, like an UWB (Ultra Wideband) probe in a near-field antenna measurement system or in a non-destructive material testing / inspection system, where a wide bandwidth provides a good measurement resolution. Furthermore, a frequency-scaled antenna might be used in the designated UWB communication bands.

\section{ACKNOWLEDGMENT}

The authors would like to thank Dr. Tim Brown from
University of Surrey for proofreading the manuscript, and the anonymous reviewer for the dedicated work of helping the authors to improve the manuscript. They also would also like to thank Mr. Tuomas Jääskö from Pulse Finland for performing the Vivaldi prototype antenna measurements.

\section{REFERENCES}

[1] J. L. Kerr, "Short Axial Length Broad-band Horns", IEEE Trans. Antennas Propag., vol. 21, no. 5, pp. 710-714, Sep. 1973.

[2] J. D. Krauss, “Antennas”, McGraw-Hill Education, 1988.

[3] C. Burns, P. Leuchtmann, R. Vahldieck, "Analysis and Simulation of a 1-18 GHz Broadband Double-Ridged Horn Antenna”, IEEE Trans. Electromagn. Compat., vol. 45, no. 1, pp. 55-60, Feb. 2003.

[4] M. Botello-Perez, H. Jardon-Aguilar, I. Ruiz, "Design and simulation of a 1 to $14 \mathrm{GHz}$ broadband electromagnetic compatibility DRGH antenna," In Proc. 2nd Int. Conf. on Electrical and Electronics Engineering, Sep. 2005, pp. 118-121.

[5] S. Soroka, "A physically compact quad ridge horn design," Int. Symp. in Proc. Antennas and Propagation Society, Jun. 1986, vol. 24, pp. 903-906.

[6] V. Rodriguez, “An Open-Boundary Quad-Ridged Guide Horn Antenna for Use as a Source in Antenna Pattern Measurement Anechoic Chamber", IEEE Ant. and Propag. Magazine, vol. 8, no. 2, April 2006, 157-160 pp.

[7] J. Qiu, Y. Suo, W. Li, "Design and Simulation of Ultra-wideband Quad-Ridged Horn Antenna", 6th International Conference on Antenna Theory and Techniques, Jan. 2007, pp. 253-255.

[8] Z. Hradecky, P. Hamouz, "Research of Circular Polarization Quality by Using Quad Ridged Horn Antenna", Proceedings of the Fourth European Conference on Antennas and Propagation (EuCAP2010), April 2010.

[9] G. Adamiuk, T. Zwick, and W. Wiesbeck, "Dual-orthogonal polarized Vivaldi antenna for ultra-wideband applications", 17th International Conference on Microwaves, Radar and Wireless Communications (MIKON 2008), 19-21 May 2008.

[10] G. Pedersen, M. Pelosi, J. Welinder, T. Jamsa, A. Yamamoto, M. Nurkkala, S. Ling, W. Schroeder W, T. Brown, "OTA test methods for multiantenna terminals", Pervasive Mobile and Ambient Wireless Communications, Springers, 2012. pp. 197-247.

[11] O. Franek, G. F. Pedersen, "Spherical Horn Array for Wideband Propagation Measurements", IEEE Trans. Antennas Propag., vol. 59, no. 7, July 2011, pp. 2654-2660.

[12] J. Zhang, E. C. Fear, and R. H. Johnston, "Cross-Vivaldi Antenna for Breast Tumor Detection”, Wiley's Microwave and Optical Technology Letters, Vol. 51, No. 2, Feb. 2009, pp. 275-280.

[13] G. Adamiuk, T. Zwick, and W. Wiesbeck, "Compact, Dual-Polarized UWB-Antenna, Embedded in a Dielectric", IEEE Trans. Antennas Propag., Vol. 58, No. 2, Feb. 2010, pp. 279-286.

[14] M. Wajih Elsallal, John C. Mather, "An Ultra-Thin, Decade (10:1) Bandwidth, Modular "BAVA" Array with Low Cross-Polarization", IEEE International Symposium on Antennas and Propagation (APSURSI), 3-8 July 2011, pp. 1980-1983.

[15] C. A. Balanis, "Modern Antenna Handbook", John Wiley \& Sons inc., USA, 2008.

[16] R. W. Kindt, W. R. Pickles, "Ultrawideband All-Metal Flared-Notch Array Radiator", IEEE Trans. Antennas Propag., vol. 58, no. 11, November 2010, pp. 3568-3575.

[17] J. B. Knorr, Slot-line transitions, IEEE Trans. Microwave Theory Tech., Vol. 22, No. 5, May 1974, pp. 548-554.

[18] CST (Computer Simulation Technology AG), “CST Studio Suite,” Suite 2014, Darmstadt, Germany.

[19] B. Shüppert, "Microstrip/slotline transitions: modeling and experimental investigation", IEEE Transactions on Microwave Theory and Techniques, Vol. 36, Iss. 8, $6^{\text {th }}$ August 1988, pp. 1272-1282.

[20] Y. Maksimovitch, V. Mikhnev, and P. Vainikainen, "Study of Effect of Corrugated Edges on the Radiation Characteristics of GPR Antenna", 18th International Conference on Microwave Radar and Wireless Communications (MIKON), 14-16 June, 2010.

[21] SATIMO, "Satimo StarGate SG64 near-field antenna measurement system". 